\section{Comparison between conventional duplex ultrasonography and the dual- gate Doppler mode for hemodynamic measurements of the carotid arteries}

\author{
Jeanne Hersant ${ }^{1}$, Pierre Ramondou', Delphine Douillet ${ }^{2}$, Stanislas Abrard', \\ Patrick Vandeputte ${ }^{1}$, François-Xavier Lapébie ${ }^{4}$, Pierre Abraham ${ }^{5,6}$, Samir Henni ${ }^{1,6}$ \\ ${ }^{1}$ Vascular Medicine, University Hospital Center, Angers; ${ }^{2}$ Emergency, University Hospital Center, \\ Angers; ${ }^{3}$ Anesthesia and Critical Care, Hospices Civils, Lyon; ${ }^{4}$ Vascular Medicine, University \\ Hospital Rangueil, Toulouse; ${ }^{5}$ Sports Medicine, University Hospital Center, Angers; ${ }^{6}$ MitoVasc \\ Institute UMR CNRS 6015/INSERM 1083, Faculty of Medicine, Angers University, Angers, France
}

Purpose: This study investigated the correlations of hemodynamic parameters measured to quantify stenosis between the gold-standard duplex ultrasonography and the dual-gate Doppler mode. Methods: Patients examined due to suspicion of carotid artery stenosis or for surveillance of known stenosis were invited to participate in this prospective single-center study. Upon acceptance, the hemodynamic characteristics of the carotid arteries were determined successively in standard duplex and dual-gate Doppler modes. The correlations between the two modes were analyzed by computing Pearson coefficients $\left(r^{2}\right)$ and Lin concordance coefficients $\left(\rho_{c}\right)$. The degree of agreement between the two methods was visualized using Bland-Altman graphical representations.

Results: The correlation between internal carotid artery peak systolic velocity measured by standard duplex ultrasonography and dual-gate Doppler mode was good $\left(r^{2}=0.642\right)$. The same high level of correlation was observed for the carotid ratio $\left(r^{2}=0.544\right)$. However, the Bland-Altman graphical representation and the Lin concordance coefficients $\left(\rho_{c}=0.75\right.$ and $\rho_{c}=0.74$ for the internal carotid artery peak systolic velocity and carotid ratio, respectively) showed that a lack of precision generated some discrepancies between the two measurement methods.

Conclusion: Although some discrepancies were observed, the hemodynamic measurements were closely correlated between the two ultrasonography modes. Therefore, the dual-gate Doppler mode may have obvious advantages over conventional ultrasonography, offering interesting development possibilities.

Keywords: Carotid arteries stenosis; Diagnostic method; Ultrasonography; Hemodynamics; Dual-gate Doppler

Key points: The dual-gate Doppler mode measurements of the internal carotid artery peak systolic velocity and carotid ratio were closely correlated to the measurements made using conventional duplex ultrasonography. The dual-gate Doppler mode may offer interesting possibilities for the diagnosis of carotid artery stenosis.

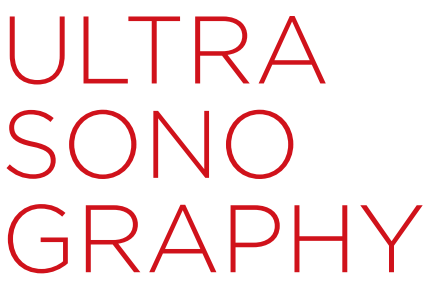

ORIGINAL ARTICLE

https://doi.org/10.14366/usg.21175 pISSN: 2288-5919 • elSSN: 2288-5943 Ultrasonography 2022;41:373-381

Received: August 20, 2021

Revised: November 8, 2021

Accepted: November 9, 2021

Correspondence to:

Samir Henni, MD, PhD, Vascular Medicine, University Hospital Center, 4 rue Larrey, 49933 Angers Cedex 9, France

Tel. +33-02-41-35-3689

Fax. +33-02-41-35-5042

E-mail: Samir.Henni@chu-angers.fr

This is an Open Access article distributed under the terms of the Creative Commons Attribution NonCommercial License (http://creativecommons.org/ licenses/by-nc/4.0/) which permits unrestricted noncommercial use, distribution, and reproduction in any medium, provided the original work is properly cited.

Copyright @ 2022 Korean Society of Ultrasound in Medicine (KSUM)

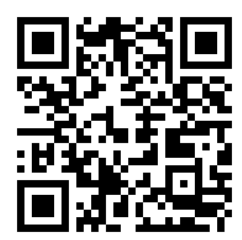

How to cite this article:

Hersant J, Ramondou P, Douillet D, Abrard S, Vandeputte $P$, Lapébie FX, et al. Comparison between conventional duplex ultrasonography and the dual-gate Doppler mode for hemodynamic measurements of the carotid arteries. Ultrasonography. 2022 Apr;41(2):373381. 


\section{Introduction}

With the global aging of the population and the subsequent increase in the number of cardiovascular events, the frequency of carotid artery stenosis (CAS) is steadily rising, with an estimated overall prevalence ranging from $1.7 \%$ to $12.5 \%$ depending on sex, age, and CAS severity [1]. Although most cases of moderate CAS remain asymptomatic and require only regular follow-up and preventive medication, severe stenosis of the internal carotid artery (ICA) can have dramatic consequences, ranging from ischemic stroke or transient ischemic attack to fatal stroke. Therefore, severe ICA stenosis ( $>70 \%$ of the artery lumen as determined following the recommendations of Sprynger et al. [2]), even if asymptomatic, is an indication for surgical intervention either by endarterectomy or stenting [3].

In recent guidelines [2], the European Society of Cardiology (ESC) recommended quantifying the percentage of ICA stenosis by a combination of the observations made in two major studies: the European Carotid Surgery Trial [4] and the North American Symptomatic Carotid Endarterectomy Trial [5]. This quantification relies on Duplex ultrasonography (DUS) determination of the peak systolic velocity (PSV) of both the ICA and common carotid artery (CCA), from which the ICA-to-CCA ratio of the PSV, namely the carotid ratio (CR), can be calculated. The take-home message of these guidelines is that stenosis should be considered hemodynamically significant when the ICA PSV $>125 \mathrm{~cm} / \mathrm{s}$ and $C R>2.0$. The degree of stenosis should be confirmed by computed tomography (CT) or magnetic resonance (MR) angiography, especially when a surgical intervention is foreseen [3]. According to these guidelines, DUS, which allows the flow velocity to be measured by combining pulsed-wave Doppler and ultrasonography (US), remains the gold standard for the first-line diagnosis and quantification of CAS. However, several technological improvements have been made available to vascular practitioners [6]. Color-DUS adds color-coded flow patterns that indicate flow direction and velocity in real time. Power Doppler color-codes the strength of the Doppler signals and is particularly well-adapted for the examination of small vessels and low-velocity flows due to its increased sensitivity. In some specific indications, contrast enhancement can be obtained through the use of ultrasound contrast agents, which mainly consist of encapsulated air bubbles [7]. eflow (Hitachi, Tokyo, Japan) and B-flow (General Electric Healthcare, Chicago, IL, USA) are high-resolution imaging modes that enhance the quality of plaque examinations. Recently, the dual-gate Doppler (DGD) mode (Hitachi) has been developed and allows the simultaneous recording of two Doppler spectra, thereby enabling simultaneous flow analysis at two distinct locations (Fig. 1).
The DGD mode has been evaluated for several applications, including obstetric cardiology $[8,9]$ and fetal vascular pathologies $[10,11]$. In adults, the DGD mode has been used in cardiology [12] and for transcranial Doppler [13-18]. However, to the best of the authors' knowledge, the DGD mode has never been used to evaluate the hemodynamics of the carotid arteries. The hypothesis of this study was that a simultaneous measurement of flow velocities at the ICA and CCA would facilitate the diagnosis of CAS, a prerequisite of which is a high level of correlation between the measurements obtained using both methods. Therefore, the objective was to assess the correlation of ICA PSV and CR measurements between the goldstandard DUS mode (two space- and time-separated reading points) and the DGD mode, which allows simultaneous measurements, in patients undergoing US examinations of the carotid arteries.

\section{Materials and Methods}

\section{Compliance with Ethical Standards}

This study was conducted in accordance with the Declaration of Helsinki and approved by the Angers University Hospital ethics committee under the reference 49RC18-0247. Patients were informed that the results of their medical examinations would be used for research purposes and could exercise their right to disagree with such use. Therefore, according to local law, written informed consent was not required.

\section{Study Population}

Data from all DUS examinations of carotid arteries performed at the Vascular Medicine Department of the authors' University Hospital Center between June 2018 and September 2019 with an Arietta V70 ultrasound platform (Hitachi) equipped with the DGD mode were used in this study. In an attempt to be as close as possible to real-life conditions, none of these examinations were excluded from the statistical analysis (e.g., technically difficult DUS due to the presence of heavily calcified plaques). In total, there were 385 US examinations, corresponding to 656 carotid arteries (271 bilateral and 114 unilateral examinations) and 375 patients as carotid artery DUS was performed on two distinct occasions for 10 patients. The main characteristics of the studied population are described in Table 1.

\section{Hemodynamic Parameters of the Carotid Arteries}

The PSVs of the ICA and CCA, as well as the CR, were determined following the guidelines published in 2018 by the ESC [2].

Briefly, patients assumed a supine position in a temperaturecontrolled room. The carotid arteries were first localized and examined for the presence of atherosclerosis plaques in B-mode with a 2-12 MHz linear probe. Thereafter, longitudinal sections of CCA 


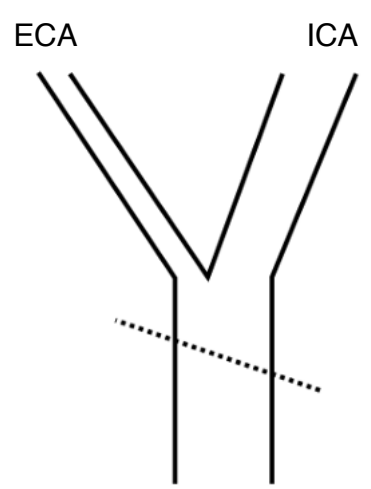

CCA

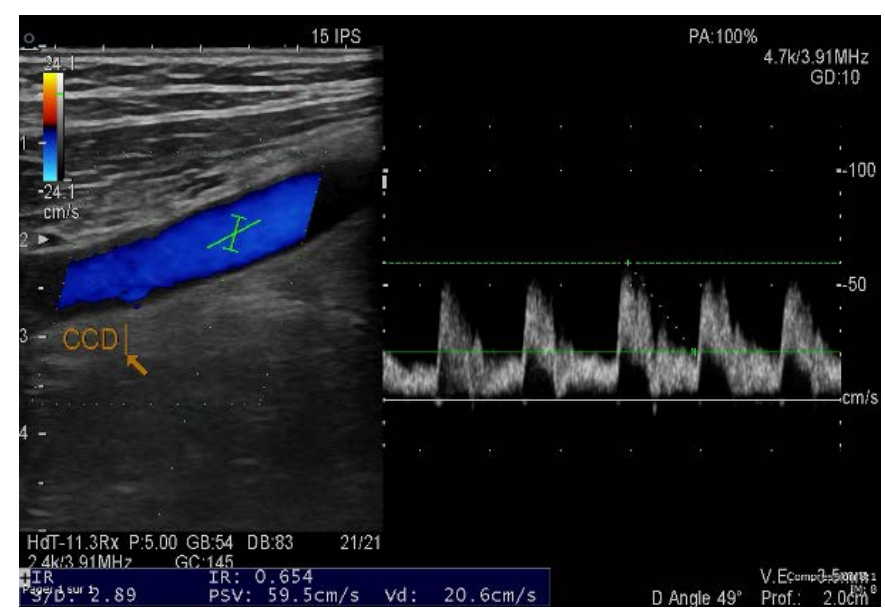

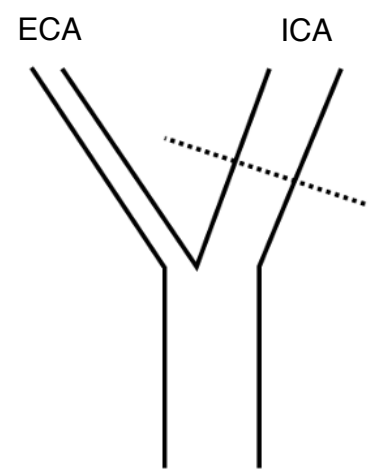

$\mathrm{CCA}$

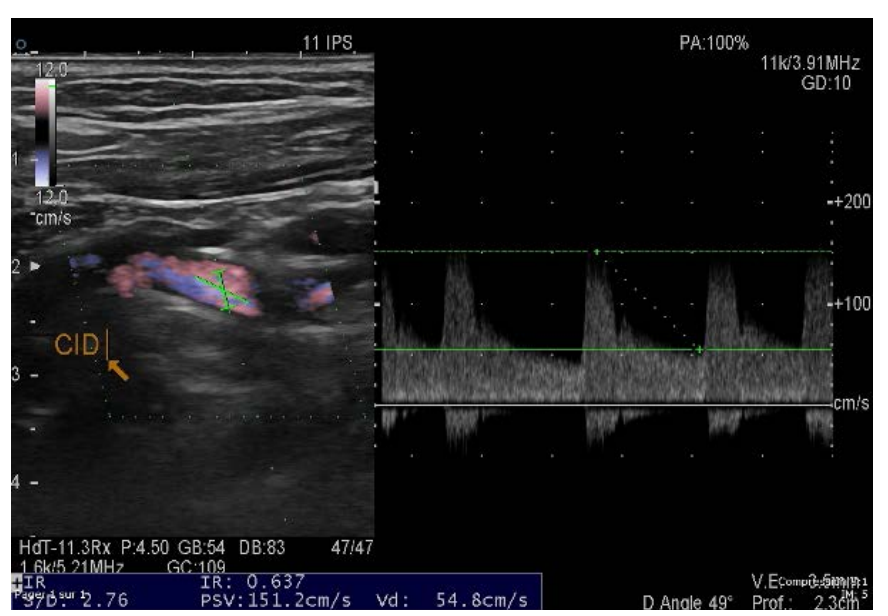

A
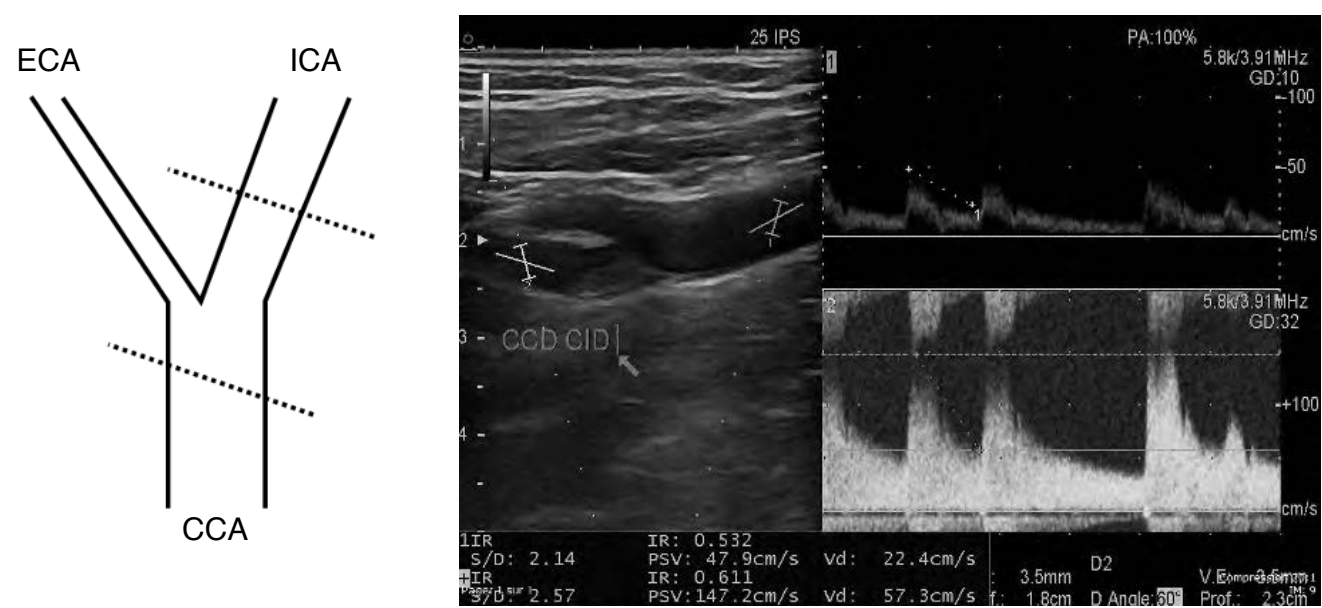

B

Fig. 1. Color-Duplex ultrasonography (DUS) versus dual-gate Doppler (DGD) measurements of carotid artery hemodynamic parameters. A. In the color-DUS gold-standard mode, two separate measurements of flow velocities, at the common carotid artery (CCA) and internal carotid artery (ICA), have to be performed. The ideal positioning of the ultrasound beam is shown by a dotted line. Typical Doppler pictures obtained in the presence of significant stenosis are given, using the CCA (left) and ICA (right) as examples. B. In DGD mode, only one recording is necessary to measure flow velocities in both the CCA and ICA since two Doppler angles can be independently adjusted to ensure a correct alignment with each blood flow. Thus, two Doppler spectra are visualized in real time. ECA, external carotid artery. 
Table 1. Study population characteristics

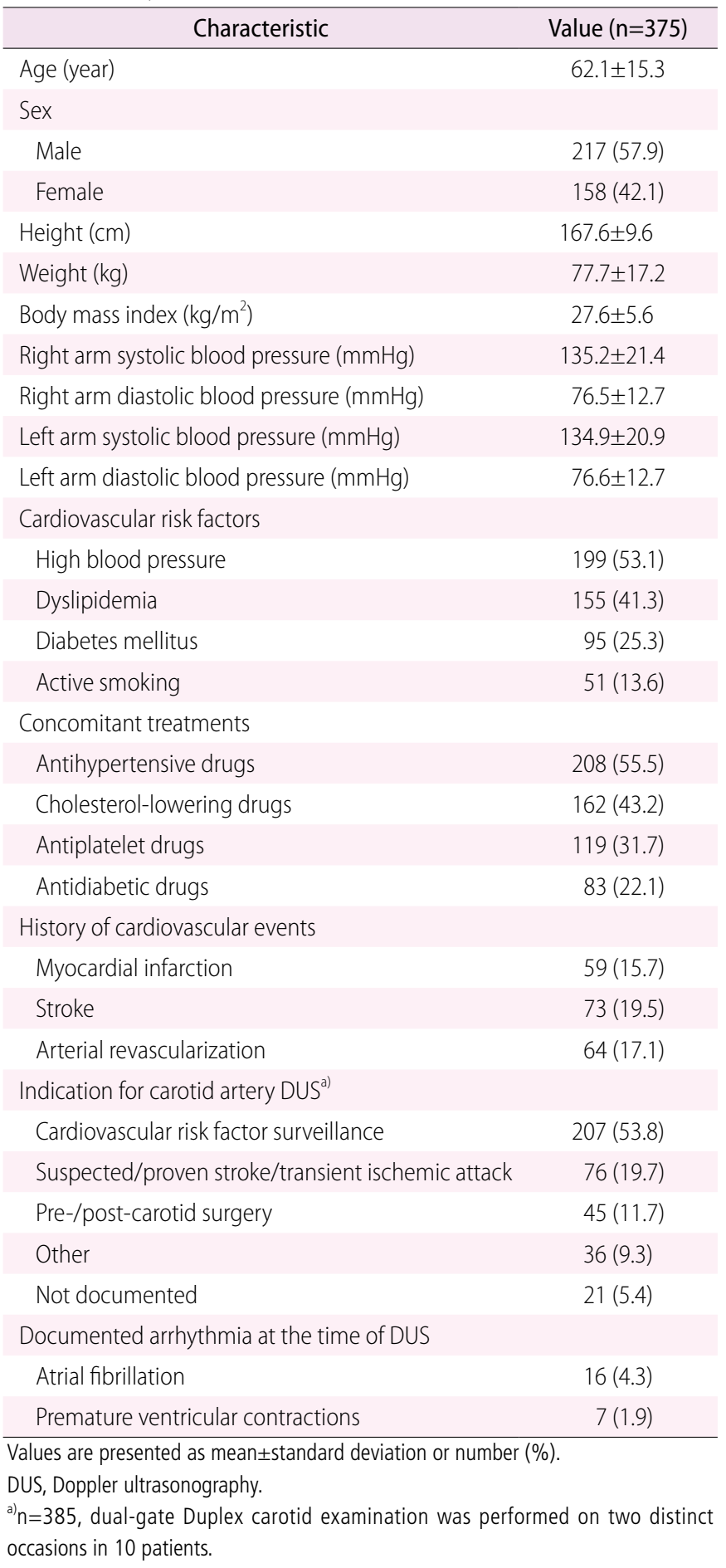

and ICA were obtained and the flows were registered, separately in the standard mode or simultaneously in the DGD mode. As far as possible, the same angulations were kept between both DUS modes. The PSV and end-diastolic velocity (EDV) were manually calculated, while the resistance index and CR were automatically computed.
For each patient, the two DUS methods were used consecutively on the same occasion. Most of the time (271 out of 385 exams), the carotid arteries were examined on both sides. Unilateral examinations of the carotid arteries were mostly performed for postsurgical purposes.

\section{Statistical Analyses}

The values of the ICA PSV and CR obtained in standard DUS mode were plotted against those obtained in DGD mode, and Pearson correlation coefficients were calculated with the Prism v8.3.0 software (GraphPad, San Diego, CA, USA). Bland-Altman graphical representations were constructed with the Prism v8.3.0 software by plotting the difference against the mean values of the ICA PSV and CR measured either in the DGD mode or in the standard DUS mode. Concordance correlation coefficients were computed according to the formula of Lin [19]. Significance was considered for P-values $<0.05$.

\section{Results}

\section{Hemodynamic Measurements}

The correlations between the measurements of ICA PSV and the calculation of $C R$ between standard DUS and DGD mode were excellent $(P<0.001)$ (Fig. $2 A, B)$. These close correlations resulted in identical conclusions in $99.4 \%$ (652/656) of cases.

However, as a close correlation does not necessarily imply good agreement in widespread datasets [20], Bland-Altman graphical representations of the data were constructed. In these representations, for each point, the difference obtained between the two US methods was plotted against the mean value of either the CR or ICA PSV (Fig. 3A, B). Overall, for both hemodynamic parameters, the agreement between the two methods was good for values close to physiological conditions; only $15.2 \%$ of the ICA PSV measurements were outside the $95 \%$ confidence interval (CI) for values under the pathological threshold $(<125 \mathrm{~cm} / \mathrm{s})$, and $3.6 \%$ of CR measurements were outside the $95 \% \mathrm{Cl}$ for values under the pathological threshold $(<2)$. These proportions of values outside the $95 \% \mathrm{Cl}$ were higher for measurements above the pathological thresholds ( $46.1 \%$ and $84.6 \%$ for the ICA PSV and CR, respectively), suggesting potential discrepancies in the diagnosis of CAS between the two US methods. Remarkably, for the ICA PSV, a bias of 6.3 $\mathrm{cm} \cdot \mathrm{s}^{-1}$ was observed in favor of the DGD mode, suggesting this velocity was overestimated as compared to the standard DUS mode (Fig. 3A).

The Bland-Altman graphical representations suggested discrepancies; however, the concordance correlation coefficients $\left(\rho_{c}\right)$ were satisfactory for both the ICA PSV $\left(\rho_{c}=0.75\right)$ and CR $\left(\rho_{c}=0.74\right)$ [21]. According to the Lin formula [22], $82.3 \%$ and $99.1 \%$ of the 
discrepancy between the two DUS methods was attributable to a lack of precision for the ICA PSV and CR, respectively.

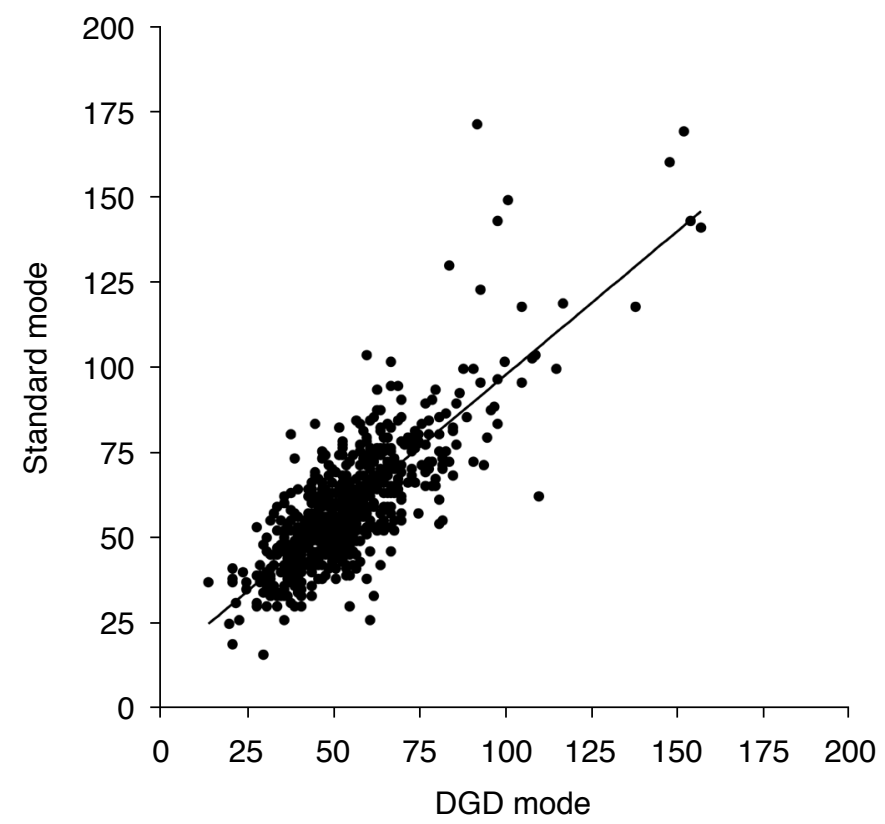

A

\section{Significant Stenoses}

Among the 656 US carotid examinations that were performed, seven resulted in the diagnosis of a significant stenosis (i.e., $>50 \%$

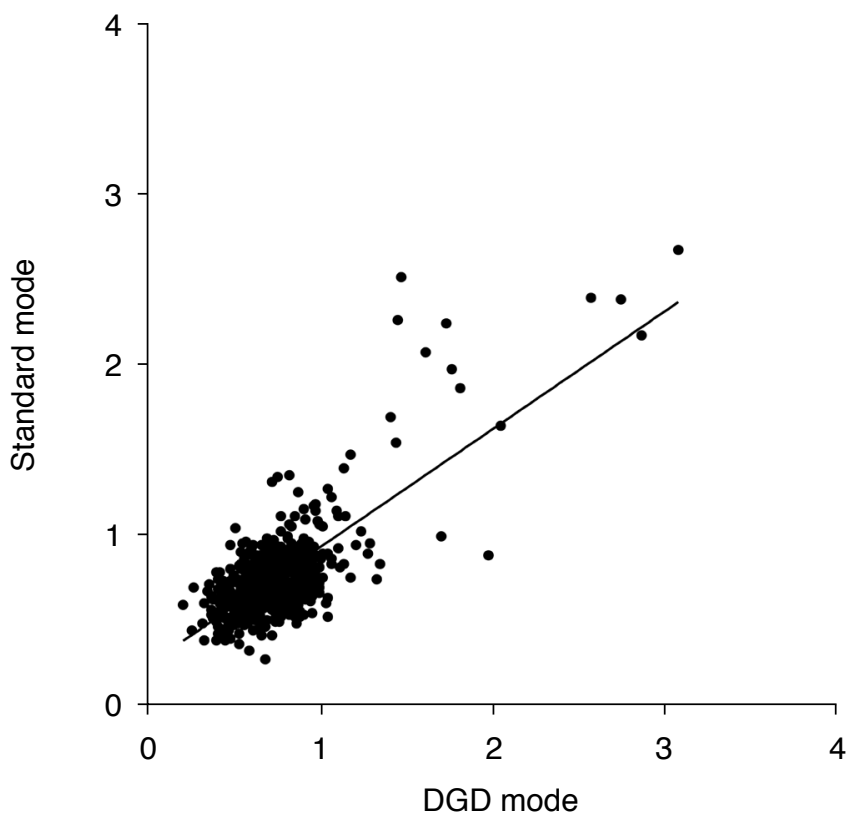

Fig. 2. Correlation analyses of hemodynamic measurements between dual-gate Doppler (DGD) and standard Duplex ultrasonography (DUS) modes.

A. The peak systolic velocity $(\mathrm{cm} / \mathrm{s})$ of the internal carotid artery measured in the DGD mode $\left(x\right.$-axis) was highly correlated $\left(r^{2}=0.642\right)$ with that measured using the standard DUS mode (y-axis). B. Likewise, the carotid ratio measurements in the DGD mode ( $x$-axis) were closely correlated $\left(r^{2}=0.544\right)$ with the values measured using the standard DUS mode (y-axis).

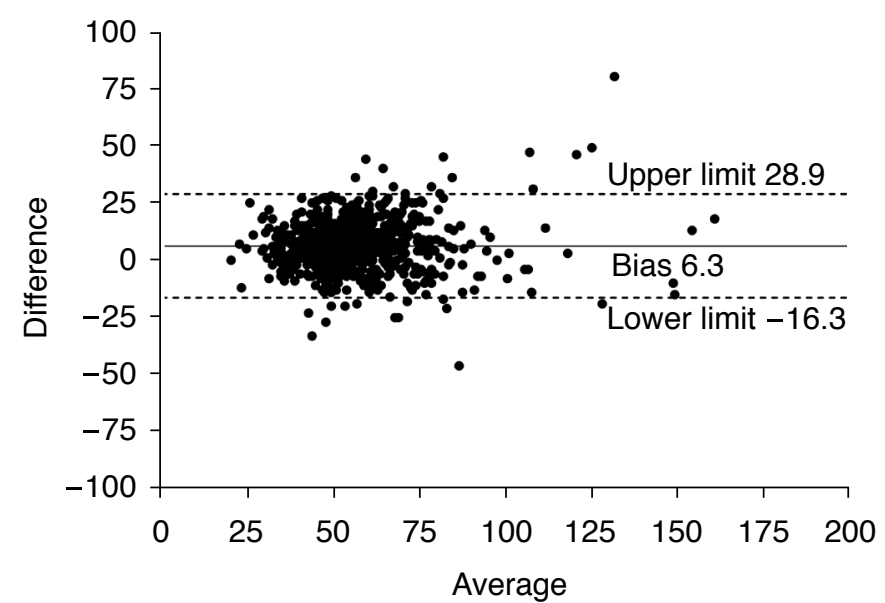

A

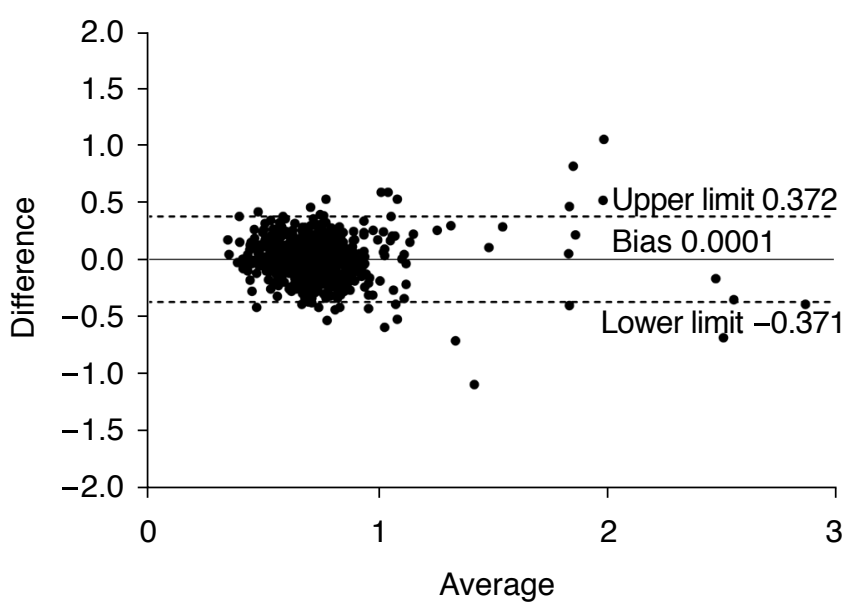

B

Fig. 3. Bland-Altman graphical representation of hemodynamic measurements.

For peak systolic velocity $(\mathrm{cm} / \mathrm{s})$ of the internal carotid artery $(A)$ and carotid ratio $(B)$, the difference obtained between standard Duplex ultrasonography (DUS) mode and the dual-gate Doppler mode was plotted against the mean of the two DUS modes. On each plot, the mean of the differences, its bias (i.e., the difference between the mean and 0 ) and its $95 \%$ confidence interval limits are indicated. Although most examinations remained between the confidence intervals for values close to physiological conditions, pathological values were highly dispersed, suggesting discrepancies between the methods regarding the diagnosis of carotid artery stenosis. 
Table 2. Hemodynamic parameters of the seven carotid stenoses diagnosed during the study determined by separate (DUS) or simultaneous measurements (DGD)

\begin{tabular}{|c|c|c|c|c|c|c|c|}
\hline \multirow{2}{*}{ Case No. } & \multicolumn{2}{|c|}{ ICA PSV $(\mathrm{cm} / \mathrm{s})$} & \multicolumn{2}{|c|}{$\mathrm{CR}$} & \multirow{2}{*}{ CT scan results } & \multirow{2}{*}{ Clinical consequences } & \multirow{2}{*}{ Medical management } \\
\hline & DUS & DGD & DUS & DGD & & & \\
\hline 1 & $141^{\text {a) }}$ & $156^{\mathrm{a})}$ & $2.39^{a)}$ & $2.56^{\mathrm{a})}$ & Complete occlusion & Ischemic stroke & Unknown $^{\text {b) }}$ \\
\hline 2 & $143^{\mathrm{a})}$ & $153^{\mathrm{a})}$ & $2.38^{\mathrm{a})}$ & $2.73^{\mathrm{a})}$ & Stenosis $50 \%$ & None & Preventive endarterectomy \\
\hline 3 & $160^{a)}$ & $147^{\mathrm{a})}$ & $2.67^{\mathrm{a})}$ & $3.06^{\mathrm{a})}$ & Stenosis 60\% & Ischemic stroke & Endarterectomy \\
\hline 4 & $143^{\mathrm{a})}$ & 97 & $2.51^{\text {a) }}$ & 1.47 & Stenosis 40\% & Ischemic stroke & Endarterectomy \\
\hline 5 & $130^{\mathrm{a})}$ & 83 & $2.24^{\mathrm{a})}$ & 1.73 & Stenosis 25\% & Ischemic stroke & Endarterectomy \\
\hline 6 & $149^{\mathrm{a})}$ & 100 & $2.07^{\mathrm{a})}$ & 1.61 & Not done & None & Unknown ${ }^{\text {b) }}$ \\
\hline 7 & 118 & $137^{\mathrm{a})}$ & 1.64 & $2.04^{\mathrm{a})}$ & Stenosis $>70 \%{ }^{c}$ & None & Preventive endarterectomy \\
\hline
\end{tabular}

The results of $\mathrm{CT}$ are indicated when available. Likewise, the clinical consequences of carotid artery stenosis, as well as its medical management, are indicated when available. DUS, Duplex ultrasonography; DGD, dual-gate Doppler; ICA, internal carotid artery; PSV, peak systolic velocity; CR, carotid ratio; CT, computed tomography.

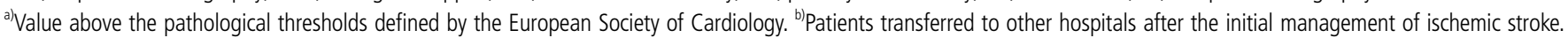

${ }^{c} \mathrm{CT}$ scan performed 2 years later, after a follow-up DUS in standard mode that yielded values above the pathological thresholds (ICA PSV=207 cm/s, CR=2.49).

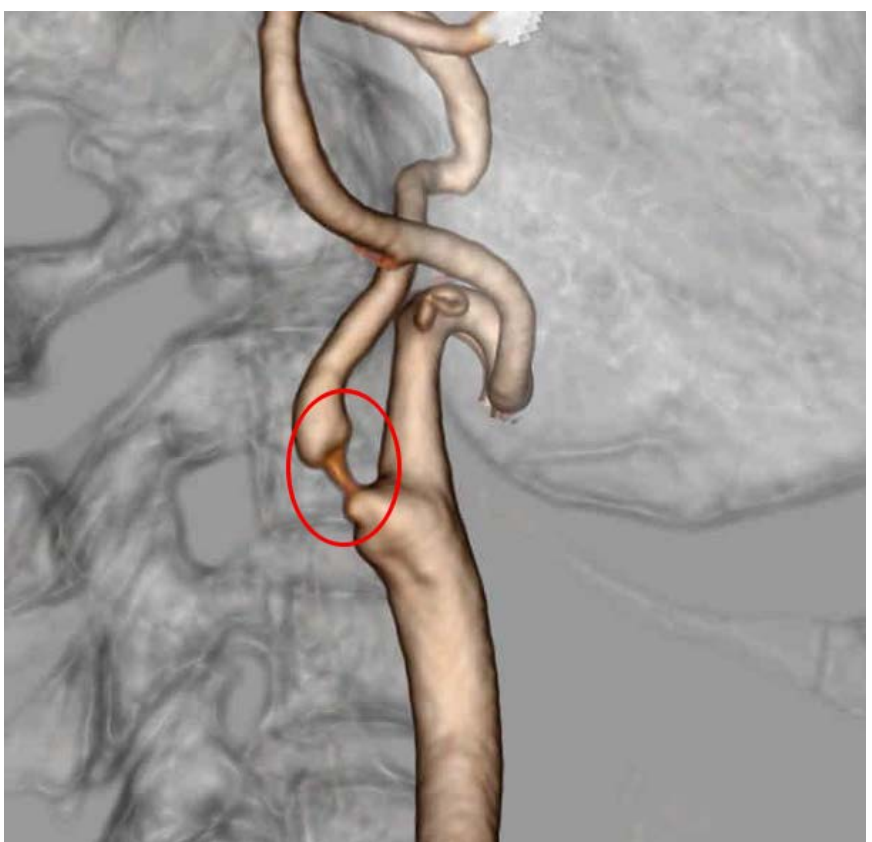

Fig. 4. Representative three-dimensional reconstruction of the results of computed tomography angiography of supra-aortic arterial trunks (corresponding to carotid artery stenosis case No. 3 of Table 1). The Duplex ultrasonography examination shows suspicion for hemodynamically significant stenosis of the right internal carotid artery (peak systolic velocity of 160 and $147 \mathrm{~cm} / \mathrm{s}$ in standard and dual-gate Doppler mode, respectively; carotid ratio of 2.67 and 3.06 in standard and dual-gate Doppler mode, respectively). The three-dimensional reconstruction shows $60 \%$ stenosis of the right internal carotid artery in its proximal portion (red ellipse). with an ICA PSV >125 cm/s and CR >2.0) (Table 2). Three were diagnosed by both US modes (case Nos. 1, 2, and 3 in Table 2). All three of these cases of CAS were further confirmed by CT scans. A representative CT scan result is illustrated in Fig. 4 (CAS case No. 3 in Table 2).

For three patients (case Nos. 4, 5, and 6 in Table 2), the stenosis was diagnosed in the standard DUS mode only. Interestingly, CAS was further confirmed by a CT scan and a subsequent endarterectomy was performed in cases Nos. 4 and 5. Thus, these two cases were false negatives of the DGD mode. Case No. 6 may also fall into this category, but the stenosis was never confirmed since no other examinations were performed.

Case No. 7 of stenosis in Table 1 was diagnosed in the DGD mode only. The standard DUS mode evaluated the stenosis as including $40 \%-49 \%$ of the ICA lumen. At the time of this DUS examination, no CT scan was performed to further confirm or rule out the presence of significant stenosis. However, DUS performed 2 years later revealed an aggravation of the ICA stenosis $(50 \%-60 \%)$, which was on this occasion confirmed by a CT scan (>70\% stenosis) and led to a preventive endarterectomy.

\section{Discussion}

During the last few decades, considerable progress has been made in the diagnosis and management of both asymptomatic and symptomatic CAS. Furthermore, recent medical studies have convincingly demonstrated the benefits of early detection and management of this pathology, especially in terms of stroke or transient ischemic attack incidence [23-27]. Due to its low cost, low risk, and broad availability, DUS remains, by far, the first-choice method for early detection of CAS. However, this examination has 
high inter- and intra-operator variability [28], and confirmatory CT or MR angiography is mandatory before any surgical intervention. In an attempt to facilitate the diagnosis of CAS by DUS, a prospective study was conducted of 656 carotid artery examinations to assess the correlation between the hemodynamic parameters measured either by the gold-standard DUS method or the DGD mode.

Overall, the correlations between the gold-standard DUS and the DGD mode for measurements of the CR and PSV of the ICA were above $99 \%$. However, both Bland-Altman graphical representations and concordance correlation coefficients suggested discrepancies between the two measurement methods, especially for values above pathological thresholds.

Accordingly, the DGD mode failed to diagnose two out of seven cases of CAS that were identified by the standard DUS mode and further confirmed by a CT scan. These false-negative results could have led to improper decision-making if the carotid arteries were examined in DGD mode only. The DGD mode also failed to diagnose a case of CAS that was significant based on the results obtained by the standard DUS mode, but was never further explored. Conversely, one significant case of CAS was diagnosed in the DGD mode but not in the standard DUS mode. A careful review of the US images determined that the measurement of CCA PSV was farther from the carotid bulb (more proximal) in the DGD mode than in the standard mode, which may explain the observed discrepancy. However, a follow-up examination performed 2 years later (only in the standard DUS mode) revealed significant stenosis that was further confirmed by a CT scan. In this case, the DGD mode allowed earlier detection of a significant CAS. Fortunately, this delayed diagnosis had no medical consequences for the patient, who remained asymptomatic until a preventive endarterectomy was performed.

The major limitation of the present study is the impossibility for the vascular practitioner to determine flow velocities at exactly the same positions in the standard DUS mode and the DGD mode. For this reason, small variations in flow velocity measurements, which are inevitable when the probe is repositioned, can cause the diagnosis to change from "non-significant" to "significant" for values close to the pathological thresholds. This may explain the discrepancies observed between the two US modes regarding CAS cases.

Although the aim of the present study was to evaluate the degree of concordance between the hemodynamic measurements made using the conventional DUS and DGD modes, another major limitation of the present results is the low number of cases of significant CAS (7, see Table 2) which precludes any robust comparison of the capacity of the two DUS modes to detect clinically significant CAS.

Another parameter that may have affected the US examination conclusions and the discrepancies observed between the two methods is possible arrhythmia. Indeed, the ICA and/or CCA PSV can be greatly affected if it is measured just after a premature ventricular contraction or a compensatory pause [29]. The DGD mode has the major advantage of allowing vascular practitioners to measure flow velocities during the same cardiac cycle, thereby obviating these issues encountered in patients with arrhythmia. As an example, for one patient with documented severe arrhythmia, the DGD mode enabled a correct diagnosis of the stenosis as non-significant, whereas the reference method yielded a CR above the pathological threshold. Furthermore, the ESC guidelines advocate determining the CR and ICA PSV, but also the ICA EDV to diagnose CAS [2]. At the authors' University Hospital Center, this last hemodynamic parameter is indirectly taken into consideration for the quantification of stenosis degree through the automated calculation of the ICA resistive index (resistive index=(PSV-EDV)/PSV). One may speculate that the integration of the recommended threshold for EDV could have decreased the number of discrepancies observed between the two DUS modes. In this regard, future validation studies may benefit from the addition of the ICA EDV into the diagnosis algorithm.

Lastly, the same ICA PSV and CR cutoff values were used as significance thresholds for both conventional DUS and the DGD mode. It remains possible that these thresholds are not adapted to DGD, and future studies will be needed to validate these values.

In conclusion, conventional DUS and the DGD mode measured the hemodynamic characteristics of carotid artery flow with excellent agreement. This good level of agreement tended to decrease for values above pathological thresholds, which were responsible for the small proportion of discordant diagnoses. Overall, the DGD mode allowed reliable measurements of carotid artery flow with the main advantage of simultaneous flow recording at ICA and CCA. One may speculate that future technical developments in US will further enhance the sensitivity and the specificity of US examinations of the carotid arteries. Given this close correlation between the reference method and the DGD mode, future studies could now be envisaged to demonstrate the potential benefits of the DGD technology in terms of clinical outcome prediction or acquisition time. The DGD mode, which is mostly used in cardiology, may also bring major benefits to other vascular investigations where flow velocity must be measured at several branches of the vasculature, such as the renal arteries.

ORCID: Jeanne Hersant: https://orcid.org/0000-0002-7126-0534; Pierre Ramondou: https://orcid.org/0000-0002-1288-8866; Delphine Douillet: https://orcid.org/00000002-6458-2965; Stanislas Abrard: https://orcid.org/0000-0002-5883-1777; Patrick Vandeputte: https://orcid.org/0000-0002-9425-9253; François-Xavier Lapébie: https://orcid.org/0000-0002-3101-3889; Pierre Abraham: https://orcid.org/00000001-7053-2801; Samir Henni: https://orcid.org/0000-0002-9838-481X 


\section{Author Contributions}

Conceptualization: Lapébie FX, Abraham P, Henni S. Data acquisition: Hersant J, Ramondou P, Henni S. Data analysis or interpretation: Hersant J, Douillet D, Abrard S, Vandeputte P, Henni S. Drafting of the manuscript: Hersant J, Vandeputte P. Critical revision of the manuscript: Hersant J, Ramondou P, Abrard S, Vandeputte P, Lapébie FX, Abraham P, Henni S. Approval of the final version of the manuscript: all authors.

\section{Conflict of Interest}

No potential conflict of interest relevant to this article was reported.

\section{Acknowledgments}

The authors would like to thank Mrs Stéphanie Maréchal-Girault and Marine Mauboussin, as well as the Maison de la Recherche Clinique of Angers University Hospital Center for technical help.

\section{References}

1. de Weerd M, Greving JP, de Jong AW, Buskens E, Bots ML. Prevalence of asymptomatic carotid artery stenosis according to age and sex: systematic review and metaregression analysis. Stroke 2009;40:1105-1113.

2. Sprynger M, Rigo F, Moonen M, Aboyans V, Edvardsen T, de Alcantara ML, et al. Focus on echovascular imaging assessment of arterial disease: complement to the ESC guidelines (PARTIM 1) in collaboration with the Working Group on Aorta and Peripheral Vascular Diseases. Eur Heart J Cardiovasc Imaging 2018;19:11951221.

3. Aboyans V, Ricco JB, Bartelink ME, Bjorck M, Brodmann M, Cohnert T, et al. 2017 ESC Guidelines on the Diagnosis and Treatment of Peripheral Arterial Diseases, in collaboration with the European Society for Vascular Surgery (ESVS): document covering atherosclerotic disease of extracranial carotid and vertebral, mesenteric, renal, upper and lower extremity arteries. Endorsed by: the European Stroke Organization (ESO) The Task Force for the Diagnosis and Treatment of Peripheral Arterial Diseases of the European Society of Cardiology (ESC) and of the European Society for Vascular Surgery (ESVS). Eur Heart J 2018;39:763-816.

4. MRC European Carotid Surgery Trial: interim results for symptomatic patients with severe (70-99\%) or with mild (0-29\%) carotid stenosis. European Carotid Surgery Trialists' Collaborative Group. Lancet 1991;337:1235-1243.

5. North American Symptomatic Carotid Endarterectomy Trial Collaborators; Barnett HJ, Taylor DW, Haynes RB, Sackett DL, Peerless SJ, et al. Beneficial effect of carotid endarterectomy in symptomatic patients with high-grade carotid stenosis. N Engl J Med 1991;325:445-453.
6. Saxena A, Ng EYK, Lim ST. Imaging modalities to diagnose carotid artery stenosis: progress and prospect. Biomed Eng Online 2019;18:66.

7. Ignee A, Atkinson NS, Schuessler G, Dietrich CF. Ultrasound contrast agents. Endosc Ultrasound 2016;5:355-362.

8. Lee MY, Won HS, Shim JY, Lee PR, Kim A, Kil EM, et al. Novel technique for measurement of fetal right myocardial performance index using dual gate pulsed-wave Doppler. J Ultrasound Med 2017;36:1585-1594.

9. Takano M, Nakata M, Nagasaki S, Ueyama R, Morita M. Assessment of diastolic function of normal fetal heart using dualgate Doppler. Ultrasound Obstet Gynecol 2018;52:238-242.

10. Ito A, Nakata M, Oji A, Takano M, Umemura N, Nagasaki S, et al. Diagnosis of umbilical cord entanglement in a monochorionic diamniotic twin pregnancy with spontaneous septostomy of the dividing membranes using dual-gate Doppler imaging. J Med Ultrason (2001) 2018;45:189-192.

11. Takahashi Y, Iwagaki S, Chiaki R, Asai K, Matsui M, Kawabata I. Ultrasonic identification of pump twin by dual-gate Doppler in a monochorionic-triamniotic triplet twin reversed arterial perfusion sequence before preventative radiofrequency ablation: a case report. J Med Ultrason (2001) 2018;45:185-187.

12. Liu J, Chen L, Zhang X, Shang Y, Wang F, Tang H. Evaluating the left ventricular diastolic function of diabetes mellitus patients using dual-gate Doppler. Sheng Wu Yi Xue Gong Cheng Xue Za Zhi 2015;32:163-167.

13. Darbellay GA, Duff R, Vesin JM, Despland PA, Droste DW, Molina $C$, et al. Solid or gaseous circulating brain emboli: are they separable by transcranial ultrasound? J Cereb Blood Flow Metab 2004;24:860-868.

14. Devuyst G, Darbellay GA, Vesin JM, Kemeny V, Ritter M, Droste DW, et al. Automatic classification of HITS into artifacts or solid or gaseous emboli by a wavelet representation combined with dualgate TCD. Stroke 2001;32:2803-2809.

15. Mess WH, Titulaer BM, Ackerstaff RG. Middle cerebral artery anatomy and characteristics of embolic signals: a dual gate computer simulation study. Ultrasound Med Biol 1999;25:531-539.

16. Mess WH, Titulaer BM, Ackerstaff RG. A new algorithm for off-line automated emboli detection based on the pseudo-wigner power distribution and the dual gate TCD technique. Ultrasound Med Biol 2000;26:413-418.

17. Smith JL, Evans DH, Fan L, Bell PR, Naylor AR. Differentiation between emboli and artefacts using dual-gated transcranial Doppler ultrasound. Ultrasound Med Biol 1996;22:1031-1036.

18. Smith JL, Evans DH, Naylor AR. Signals from dual gated TCD systems: curious observations and possible explanations. Ultrasound Med Biol 1997;23:15-24.

19. Lin LI. A concordance correlation coefficient to evaluate reproducibility. Biometrics 1989;45:255-268. 
20. Bland JM, Altman DG. Statistical methods for assessing agreement between two methods of clinical measurement. Lancet 1986;1:307310.

21. Partik BL, Stadler A, Schamp S, Koller A, Voracek M, Heinz G, et al. $3 D$ versus $2 D$ ultrasound: accuracy of volume measurement in human cadaver kidneys. Invest Radiol 2002;37:489-495.

22. Lin LI. Total deviation index for measuring individual agreement with applications in laboratory performance and bioequivalence. Stat Med 2000;19:255-270.

23. Hadar N, Raman G, Moorthy D, O'Donnell TF, Thaler DE, Feldmann $E$, et al. Asymptomatic carotid artery stenosis treated with medical therapy alone: temporal trends and implications for risk assessment and the design of future studies. Cerebrovasc Dis 2014;38:163173.

24. Halliday A, Harrison M, Hayter E, Kong X, Mansfield A, Marro $J$, et al. 10-year stroke prevention after successful carotid endarterectomy for asymptomatic stenosis (ACST-1): a multicentre randomised trial. Lancet 2010;376:1074-1084.

25. Naylor AR, Sillesen $H$, Schroeder TV. Clinical and imaging features associated with an increased risk of early and late stroke in patients with symptomatic carotid disease. Eur J Vasc Endovasc Surg 2015;49:513-523.

26. Seiden SW. Endarterectomy for asymptomatic carotid artery stenosis. JAMA 1995;274:1506.

27. Rothwell PM, Eliasziw M, Gutnikov SA, Fox AJ, Taylor DW, Mayberg $M R$, et al. Analysis of pooled data from the randomised controlled trials of endarterectomy for symptomatic carotid stenosis. Lancet 2003:361:107-116.

28. Kohler T, Langlois Y, Roederer GO, Phillips DJ, Beach KW, Primozich $\mathrm{J}$, et al. Sources of variability in carotid duplex examination: a prospective study. Ultrasound Med Biol 1985;11:571-576.

29. Tahmasebpour HR, Buckley AR, Cooperberg PL, Fix CH. Sonographic examination of the carotid arteries. Radiographics 2005;25:15611575. 